\title{
Identifying Technical Procedures in Pulmonary Medicine That Should Be Integrated in a Simulation-Based Curriculum: A National General Needs Assessment
}

\author{
Leizl Joy Nayahangan ${ }^{\mathrm{a}}$ Paul Frost Clementsen ${ }^{\mathrm{a}, \mathrm{b}} \quad$ Charlotte Paltved $^{\mathrm{c}}$ \\ Karen Gilboe Lindorff-Larsen ${ }^{d}$ Bjørn Ulrik Nielsen ${ }^{e}$ Lars Konge $^{a}$ \\ ${ }^{a}$ Copenhagen Academy for Medical Education and Simulation, University of Copenhagen and The Capital Region \\ of Denmark, Copenhagen, ${ }^{b}$ Department of Respiratory Medicine, Gentofte University Hospital, Hellerup, \\ 'MidtSim - Centre for Human Resources, Central Region of Denmark and Aarhus University, Aarhus, ${ }^{\mathrm{d}}$ NordSim - \\ Centre for Skills Training and Simulation, Aalborg University Hospital, Aalborg, and ${ }^{\mathrm{S}} \mathrm{Sim}-\mathrm{C}$ - the Simulation Centre \\ of Odense University Hospital, Odense, Denmark
}

\section{Key Words}

Needs assessment · Curriculum development · Simulation ·

Pulmonary medicine $\cdot$ Simulation-based education

\begin{abstract}
Background: Simulation training is a revolutionary addition to health care education. However, developing simulationbased training programs is often dictated by those simulators that are commercially available. Curriculum development requires deliberate planning and a standardized approach, including a 'general needs assessment'. Objectives: The aim of this study was to perform a national general needs assessment to identify technical procedures in pulmonary medicine that should be integrated in a simulationbased curriculum. Methods: A three-round Delphi process was initiated among 62 key opinion leaders. Round 1 was an open-ended question to identify technical procedures pulmonologists should learn. Round 2 was a survey using a newly developed needs assessment formula to explore the fre-
\end{abstract}

quency of procedures, number of operators, risk or discomfort when performed by an inexperienced doctor, and feasibility of simulation-based training. In round 3, results were reviewed and ranked according to priority. Results: The response rates for the three rounds were 74, 63, and $60 \%$, respectively. The Delphi process reduced the 30 procedures identified in round 1 to 11 prioritized technical procedures in round 3. These were: flexible bronchoscopy, pleurocentesis, endobronchial ultrasound, endoscopic ultrasoundguided fine-needle aspiration, noninvasive ventilation treatment, transthoracic biopsy of pleural or lung tumor, focused ultrasound scanning of the lungs, chest tube insertion, needle biopsy of visible lymph node/tumor of the skin, focused ultrasound scanning of the heart, and thoracoscopy. Conclusion: We performed a Delphi study using a needs assessment formula, which identified 11 technical procedures that are highly suitable for simulation-based training. Medical educators can use this list as a resource in planning simulation-based training programs for trainees in pulmonary medicine.

\section{KARGER}

E-Mail karger@karger.com

www.karger.com/res
(C) 2016 S. Karger AG, Basel

0025-7931/16/0916-0517\$39.50/0 


\section{Introduction}

The current training approach for future specialists in pulmonary medicine follows the traditional apprenticeship program, in which trainees learn by shadowing and directly observing their supervisors. This classical approach faces a number of challenges, including work hour restriction for trainees, limited exposure to the procedures, a lack of facilities and a lack of skilled supervisors, and, most importantly, rising concerns towards patient safety $[1,2]$. Consequently, it is inevitable that the apprenticeship model will have to be restructured and reformed $[3,4]$. One of the most important focus areas in the last two decades in response to this need is the use of simulation-based education to train technical skills. There is growing evidence that skills acquired in a simulation-based environment can be directly transferred to improved patient care practices and better outcomes [5].

Simulation training has become increasingly popular and should be considered an integral thread in the delivery of medical education [3]. However, the integration of simulation-based education into the curricula has been challenging and is often based on experiential notions rather than following a standardized approach [3]. Unfortunately, the development of simulation-based training programs is often dictated by what simulation equipment is available commercially. According to Kern et al. [6], developing a curriculum follows a six-step approach in which the first vital step is problem identification and needs assessment. Linking the medical curricula to contemporary health care needs is crucial. A general needs assessment aims to address the problems that have been identified and bridge the gaps between the current approach and an ideal approach.

The aim of our study was to conduct a national needs assessment to identify technical procedures in pulmonary medicine that should be integrated in a simulation-based curriculum.

\section{Materials and Methods}

A Delphi technique was used to identify the technical procedures. Developed in the 1950s, the Delphi process is a structured approach that is widely accepted in terms of achieving convergence of opinion from a group of experts concerning a specific topic [7].

\section{Research Steering Group}

The general needs assessment research steering group consisted of three members: a nurse who specializes in health care management and is also the coordinator of the Simulation Centre at
Rigshospitalet [8], a consultant in pulmonary medicine with many years of experience in simulation-based education, and a thoracic surgeon who is also an associate professor and the head of the Simulation Centre. All members have been involved in developing courses across different specialties and offering training opportunities for doctors to practice different clinical skills in a simulationbased environment. The steering group supervised, monitored, and managed all processes in the study, including identification of participants, analysis of data, and creation of subsequent questionnaires.

\section{Study Population}

The participants in the study were identified through the national educational program for pulmonary medicine, the Danish Health and Medicines Authority, and the Danish Society of Respiratory Medicine. All regions of Denmark were represented. A total of 62 participants were identified and characterized as follows: postgraduate clinical associates $(n=3)$, head of pulmonary departments across all regions $(n=22)$, head of clinical education in pulmonary medicine in each department $(n=16)$, doctors in charge of the mandatory courses in the education of respiratory physicians $(n=11)$, board of directors: Danish Society for Respiratory Medicine $(n=5)$, and board of directors: Association of Junior Pulmonary Physicians $(n=5)$.

\section{Data Collection}

The study was performed from July to November 2015 and was designed as a three-round Delphi iterative process to collect information from the participants. E-mails and telephone contacts were used for communication.

\section{Round 1 - Brainstorming}

The first round of the Delphi process was a brainstorming phase in which the participants were asked to identify what technical procedures trainees should be able to perform and be competent in before becoming specialists in pulmonary medicine. For the purposes of the study, technical procedures were defined as clinical procedures that were characteristically specific to pulmonary medicine. During this round, an extensive list of 68 different procedures including nontechnical skills was identified. The list was reviewed and reduced, thereby eliminating procedures that were deemed nontechnical (for example, difficult conversations and other communication skills).

Round 2 - Survey Using a Needs Assessment Formula We developed a formula called the Copenhagen Academy for Medical Education and Simulation (CAMES) needs assessment formula to systematically explore and assess the training needs of upcoming pulmonologists. The formula is described below:

Need for simulation-based training $=$ Frequency of procedure $\times$ Number of physicians that should be able to perform the procedure $\times$ Discomfort and/or risk for patients if the procedure is performed by an inexperienced doctor $\times$ Feasibility of learning the procedure in a simulation-based environment.

The technical procedures that were identified in round 1 were included in a survey consisting of the following two questions: (1) How frequently is the procedure performed in your department every year? (2) How many doctors should learn to perform the procedure in your department? 
Fig. 1. Overview of the Delphi process.

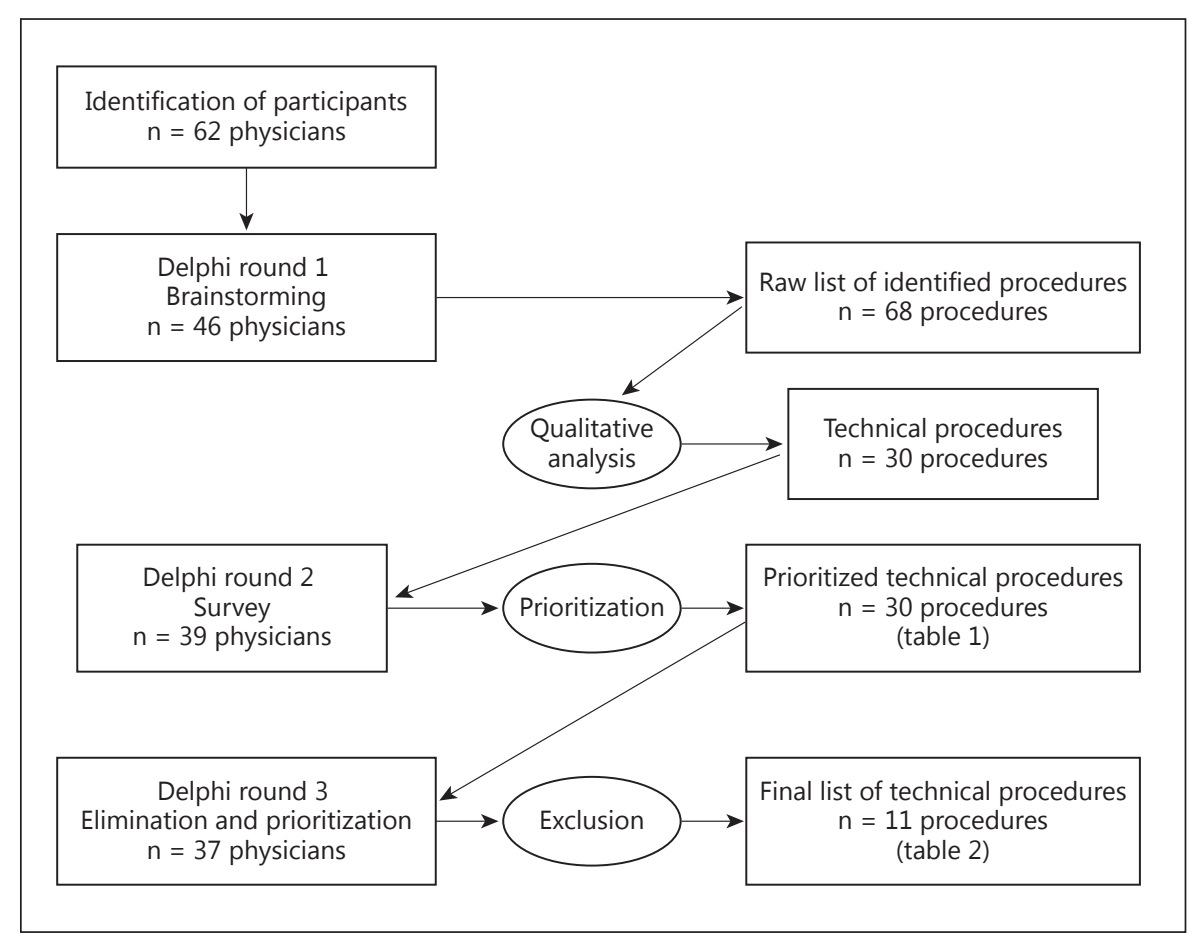

The statement 'The procedure is very uncomfortable and/or risky to patients when performed by an inexperienced doctor' was explored on a five-point Likert scale anchored by degree of agreement with 1 as the highest (strongly agree) and 5 as the lowest (strongly disagree).

Feasibility was assessed by the steering group using a five-point Likert scale. Each procedure was rated according to its suitability to be offered in a simulation-based environment, equipment availability, and the costs associated with developing and running it as a simulation-based training program.

The survey was developed in SurveyXact (Rambøll Management Consulting, Copenhagen, Denmark).

Round 3 - Elimination and Prioritization

In round 3 , the prioritized list of technical procedures from round 2 was sent to the participants for review and they either agreed with the ranking or revised it according to their own opinion. They were advised to answer ' 0 ' if they felt that the procedure was not a priority to be taught in a simulation-based environment. It was predetermined that a procedure item would be eliminated if more than one third of the respondents answered ' 0 '. Figure 1 shows an overview of the Delphi technique used.

\section{Statistical Analysis}

The responses to the open-ended question in round 1 were analyzed qualitatively by the research steering group using a summative approach to content analysis. Descriptive analysis was used in rounds 2 and 3 . The ranked list at the end of round 2 was developed by multiplying the mean scores of each question in the CAMES needs assessment formula. In round 3, the frequency of the ranking provided for each procedure was calculated. Mean scores were calculated to achieve a prioritized list of 11 procedures in this round. Correlation analysis using Spearman's rho was performed to explore the change in the order of ranking of the included items in rounds 2 and 3.

All statistical calculations were performed using IBM SPSS (Statistical Package for Social Sciences Version 22, Chicago, Ill., USA).

\section{Results}

The response rate in round 1 was $74 \%$ (46 of 62 participants responded). An inclusive list of 30 technical procedures was identified. Round 2 of the Delphi process had a response rate of $63 \%$ (39 of 62 participants). The technical procedures were assessed using the CAMES needs assessment formula. The mean scores for each procedure were calculated and were ranked in descending order (table 1).

The response rate in round 3 was $60 \%$ (37 out 62 ). The participants provided their own ranking and 19 procedures were eliminated after frequency analysis. A total of 11 technical procedures in pulmonary medicine were included in the list after the final round of prioritization (table 2). The final list was ranked in order of high priority. 
Table 1. Thirty identified technical procedures arranged according to priority

\begin{aligned} \hline Rank & Technical procedure \\ \hline 1 & Pleurocentesis \\ 2 & Flexible bronchoscopy \\ 3 & NIV treatment \\ 4 & Placement of bladder catheter \\ 5 & Nasogastric intubation \\ 6 & Lung function test: spirometry \\ 7 & Tracheal suctioning \\ 8 & EBUS-TBNA \\ 9 & Lumbar puncture \\ 10 & EUS-FNA and EUS-B-FNA \\ 11 & Transthoracic biopsy of pleural or lung tumor \\ 12 & Focused ultrasound scanning of the lungs \\ 13 & Skin prick test \\ 14 & Needle biopsy of visible lymph node/tumor of the skin \\ 15 & Ascites puncture \\ 16 & Mannitol/methacholine provocation \\ 17 & Six-minute walk test \\ 18 & Chest tube insertion \\ 19 & Mantoux test \\ 20 & Focused ultrasound scanning of the heart \\ 21 & Exhaled nitric oxide test \\ 22 & Allergen provocation test \\ 23 & Allergen immunotherapy \\ 24 & Ultrasound-guided arterial puncture \\ 25 & Ergospirometry \\ 26 & Pleurodesis \\ 27 & Ultrasound-guided venous access \\ 28 & Thoracoscopy \\ 29 & Eucapnic hyperventilation test \\ 30 & Continuous laryngoscopic exercise test \\ \hline & \end{aligned}

Table 2. Final list of technical procedures in pulmonary medicine

\begin{tabular}{cl}
\hline Rank & Technical procedure \\
\hline 1 & Flexible bronchoscopy \\
2 & Pleurocentesis \\
3 & EBUS-TBNA \\
4 & EUS-FNA/EUS-B-FNA \\
5 & NIV treatment \\
6 & Transthoracic biopsy of pleural or lung tumor \\
7 & Focused ultrasound scanning of the lungs \\
8 & Chest tube insertion \\
9 & Needle biopsy of visible lymph node/tumor of the skin \\
10 & Focused ultrasound scanning of the heart \\
11 & Thoracoscopy \\
\hline
\end{tabular}

There was a strong positive correlation between the order of included items from round 2 (CAMES needs assessment formula) and round 3 (ranking according to participants), with a Spearman's rank correlation coefficient of $r=0.96$ and a $p$ value of $<0.001$. This is highly significant indicating a minor change in the order of prioritization of the technical procedures.

\section{Discussion}

We conducted a nationwide needs assessment using the classical Delphi technique to identify training needs of future specialists in pulmonary medicine. The use of this technique is ideal for pooling ideas and information from different experts in pulmonary medicine to establish consensus [7]. To our knowledge, the present study is the first nationwide needs assessment to represent an entire medical specialty.

Simulation training has been a revolutionary addition to the current shift from traditional teaching to competence-based health care education $[9,10]$. The use of virtual reality simulation is proven to have large effects for outcomes of knowledge, skills, and behaviors [11]. Pulmonary medicine is a rapidly evolving field that shows significant changes and has adapted simulation-based education with the goal to implement uniform training curricula $[4,10]$.

The study revealed 11 technical procedures that should be integrated in a simulation-based curriculum. The highest priority was given to flexible bronchoscopy. Even though it is a safe procedure [12], the rate of complications increases when the procedure is performed by a novice operator $[13,14]$. It is suggested that residents must be sufficiently trained in order to increase the success rate of the procedure and to maximize patient comfort, patient safety, and yield [15]. Virtual reality simulators in bronchoscopy have been around for many years and are recognized as important aids in the training of both basic and diagnostic bronchoscopy [16]. To measure and assess bronchoscopy skills in a standardized manner, the use of a reliable and valid assessment tool to measure competence has been proposed $[15,17,18]$.

A more complex and specialized procedure identified in the present study is endobronchial ultrasound-guided transbronchial needle aspiration (EBUS-TBNA). Studies have found that the quality and safety of performing an EBUS-TBNA is dependent on the skills and experience of the operator [4, 19]. The current European guidelines 
recommend that trainees in endosonography must follow a structured training curriculum that stresses practicing in a simulation-based environment before engaging in supervised practice on patients [4]. Endobronchial ultrasound simulators are commercially available and have been proven to provide a safe training ground for respiratory trainees to acquire the necessary skills and competences $[20,21]$. A validated tool to assess competence in EBUS-TBNA was developed in a randomized control study called the EBUS Assessment Tool (EBUSAT). The EBUSAT has an established credible pass/fail standard and is recommended to be used for certification. The study has shown that simulation-based training is more effective than the traditional apprenticeship in the initial part of the learning curve [19].

In the case of endoscopic ultrasound-guided fine-needle aspiration biopsy (EUS-FNA) and endoscopic ultrasound with bronchoscope-guided fine-needle aspiration biopsy (EUS-B-FNA), no virtual reality simulators are available in the market yet [22]. The fact that this procedure ranks fourth on our list could spur collaboration with medical simulation companies and develop a simulation-based training program to satisfy this need.

Moving down the ranked list, some of the identified procedures are basic technical skills that young doctors must learn in the early start of their residency training program. These include pleurocentesis, noninvasive ventilation treatment, and chest tube insertion. Focused ultrasound of the heart and lungs is also on the list. Ultrasound has become a versatile medical tool in pulmonary medicine and is used not only for diagnostic purposes but also as guidance for several therapeutic interventions such as punctures or drainages [23]. Studies have shown that its effectivity is directly related to the skills and experience of the operator, which means that continuous training in ultrasonography is vital. There are many commercially available ultrasound simulators making it feasible to set up simulation-based training courses in ultrasonography to optimize and meet educational needs [24].

An important part of the study was to explore the validity of the CAMES needs assessment formula. Deliberate selection of the most suitable procedures should guide the development of new training modalities. Furthermore, it is imperative that careful planning, including a systematic needs assessment, is initiated among the end users of simulation-based training. This makes it possible to plan educational activities that fit the current needs and live up to the demands of the society. Using the needs assessment formula has enabled us to methodically un- derstand the needs of trainees in pulmonary medicine and to establish a systematic approach towards curriculum development. Future studies in other specialties will hopefully confirm the usability of the CAMES needs assessment formula.

The present study has certain limitations. One limitation is the fact that the needs assessment was performed on a national level. However, we ensured that the participants were distributed geographically and that all three regions of Denmark were represented. We hope that the use of this standardized approach to perform needs assessment will inspire both regional and international interests. Another inherent limitation is the use of a webbased survey. Online surveys are effective and cost-efficient; however, one of the disadvantages is selection bias. To reduce selection bias, we included key opinion leaders from all pulmonary departments in Denmark. The high response rate in the first round could be due to the openended approach. The response rates in rounds 2 and 3 decreased, which could be due to the time-consuming nature of the survey. However, the dropout has not distorted the distribution of participants geographically, and we ensured that every pulmonary department in the country was represented. This ensured that the data gathered in all three rounds were attained at a national level. It is important to acknowledge that there might be national differences in the inclusion and prioritization of the procedures, e.g. some countries do not believe that respiratory physicians should perform EUS-FNA. It would be interesting to repeat the Delphi process in other countries and compare the results.

\section{Conclusion}

The performance of a general needs assessment is imperative in curriculum development. We performed a national Delphi process using a needs assessment formula to identify a final list of 11 technical procedures that should be integrated in a simulation-based curriculum for pulmonary medicine. The prioritized list of technical procedures may be used by medical educators as a resource in planning simulation-based training programs for doctors specializing in pulmonary medicine. 


\section{References}

$\checkmark 1$ Reznick RK, MacRae H: Teaching surgical skills - changes in the wind. N Engl J Med 2006;355:2664-2669.

$\checkmark 2$ Medford AR: Impact of the European Working Time Directive on specialty training. Qual Saf Health Care 2008;17:79-80.

$\checkmark 3$ Khamis NN, et al: A stepwise model for simulation-based curriculum development for clinical skills, a modification of the six-step approach. Surg Endosc 2016;30:279-287.

$\checkmark 4$ Vilmann P, et al: Combined endobronchial and oesophageal endosonography for the diagnosis and staging of lung cancer. European Society of Gastrointestinal Endoscopy (ESGE) Guideline, in cooperation with the European Respiratory Society (ERS) and the European Society of Thoracic Surgeons (ESTS). Eur Respir J 2015;46:40-60.

$\checkmark 5$ McGaghie WC, et al: Does simulation-based medical education with deliberate practice yield better results than traditional clinical education? A meta-analytic comparative review of the evidence. Acad Med 2011;86:706711.

6 Kern DE, Thomas PA, Hughes MT: Curriculum Development for Medical Education: A Six-Step Approach, ed 2. Baltimore, Johns Hopkins University Press, 2009, pp 5-24.

7 Hsu C-C, Sandford BA: The Delphi technique: making sense of consensus. Practical assessment, research and evaluation 2007; 12: $1-8$.
8 Konge L, et al: The Simulation Centre at Rigshospitalet, Copenhagen, Denmark. J Surg Educ 2015;72:362-365.

$\checkmark 9$ Motola I, et al: Simulation in healthcare education: a best evidence practical guide. AMEE Guide No. 82. Med Teach 2013;35:e1511e1530.

10 Fielding DI, Maldonado F, Murgu S: Achieving competency in bronchoscopy: challenges and opportunities. Respirology 2014;19:472482.

11 Cook DA, et al: Technology-enhanced simulation for health professions education: a sys tematic review and meta-analysis. JAMA 2011;306:978-988.

12 Du Rand IA, et al: British Thoracic Society guideline for diagnostic flexible bronchoscopy in adults: accredited by NICE. Thorax 2013;68(suppl 1):i1-i44.

13 Ouellette DR: The safety of bronchoscopy in a pulmonary fellowship program. Chest 2006 130:1185-1190.

14 Stather DR, et al: Trainee impact on advanced diagnostic bronchoscopy: an analysis of 607 consecutive procedures in an interventional pulmonary practice. Respirology 2013;18: 179-184.
15 Konge L, et al: Using performance in multiple simulated scenarios to assess bronchoscopy skills. Respiration 2011;81:483-490.

16 Konge L, et al: Virtual reality simulation of basic pulmonary procedures. J Bronchology Interv Pulmonol 2011;18:38-41.

17 Konge L, et al: Reliable and valid assessment of clinical bronchoscopy performance. Respiration 2012;83:53-60.

18 Konge L, et al: Establishing pass/fail criteria for bronchoscopy performance. Respiration 2012;83:140-146.

19 Konge L, et al: Simulator training for endobronchial ultrasound: a randomised controlled trial. Eur Respir J 2015;46:1140-1149.

20 Stather DR, et al: Assessment and learning curve evaluation of endobronchial ultrasound skills following simulation and clinical training. Respirology 2011;16:698-704.

21 Konge L, et al: Using virtual-reality simulation to assess performance in endobronchial ultrasound. Respiration 2013;86:59-65.

22 Konge L: Training and certification in interventional pulmonology 2015-2016. http:// www.ersbuyersguide.org/articles/current-issue-2015-2016/item/training-and-certification-in-interventional.

23 Herth FJF, Kreuter M: Ultrasound in pulmonology. Respiration 2014;87:1-2.

$\checkmark 24$ Konge L, Albrecht-Beste E, Nielsen MB: Virtual-reality simulation-based training in ultrasound. Ultraschall Med 2014;35:95-97. 